\title{
Polycyclic Aromatic Hydrocarbon (PAHs) Concentrations in Some Aquatic Macrophytes in Hilla River, Iraq
}

\author{
Fikrat M. Hassan"1, Jasim M. Salman², Ali A. Dou Abul ${ }^{3 *}$, Atheer S. Naji ${ }^{4}$ \\ ${ }^{1}$ College of Science for Woman, University of Baghdad, Baghdad, Iraq \\ ${ }^{2}$ College of Science, University of Babylon, Babylon, Iraq \\ ${ }^{3}$ Marin Science Center, University of Basrah, Basrah, Iraq \\ ${ }^{4}$ College of Ecology, Al-Qasim Green University, Babylon, Iraq \\ Email: *adouabul@mscbasra.org
}

Received 21 December 2015; accepted 19 February 2016; published 22 February 2016

Copyright (C) 2016 by authors and Scientific Research Publishing Inc.

This work is licensed under the Creative Commons Attribution International License (CC BY). http://creativecommons.org/licenses/by/4.0/

(c) (i) Open Access

\begin{abstract}
Phragmites australis, Potamogeton pectinatus, Potamogeton perfoliatus and Ceratophyllum demersum were selected to study concentrations of PAHs in lotic ecosystems. Six sampling sites were selected along Al-Hilla River and sampling was conducted in 2010 and 2011. Sixteen PAHs listed as priority pollutants were detected in the samples collected, including Naphthalene (Nap), Acenaphthylene (Acpy), Acenaphthene (Acp), Fluorene (Flu), Phenanthrene (Phen), Anthracene (Ant), Fluoranthene (Flur), Pyrene (Py), Benzo (a) Anthracene (B(a)A), Chrysene (Chry), Benzo (b) Fluoranthene (B(b)F), Benzo (k) Fluoranthene (B(k)F), Benzo (a) Pyrene (B(a)P), Dibenzo (a, h) Anthracene (D(b)A), Benzo (ghi) Perylene (B(ghi)P) and Indeno (1,2,3-cd) Pyrene (Ind). The results of the study illustrate that the PAH concentration in macrophytes varies among their species. These variances were as follows: $P$. australis 0.425 to $299.424 \mu \mathrm{g} / \mathrm{g}$ dry weight (Dw) for B(ghi)P and $\mathrm{B}(\mathrm{b}) \mathrm{F}$, respectively; $P$. perfoliatus 0.354 to $235.84 \mu \mathrm{g} / \mathrm{g}$ Dw for $\mathrm{B}(\mathrm{b}) \mathrm{F}$ and $\mathrm{B}(\mathrm{ghi}) \mathrm{P}$, respectively; $C$. demersum 0.996 to $162.942 \mu \mathrm{g} / \mathrm{g}$ Dw for Ant and B(ghi)P, respectively; and P. pectinatus 0.383 to $99.87 \mu \mathrm{g} / \mathrm{g}$ Dw for Ant and Nap, respectively. The accumulation potential of PAHs was also investigated by calculating the Bioconcentration Factor (BCF) and Bio-sediment Accumulation Factor (BSAF). The ranges of BCF ratios were 0.05 to $5334.5,0.08$ to $1602.5,0.01$ to $536.6,0.16$ to 1882 in $P$. australis, $P$. perfoliatus, $P$. pectinatus and $C$. demersum, respectively. The range of BSAF ratios were 3.14 to 1041.6 and 1.5 to 2920.8 in $P$. australis and $P$. perfoliatus, respectively.
\end{abstract}

\section{Keywords}

PAH, Macrophytes, Lotic Ecosystems, Al-Hilla River, Pollution, PAH Origin

\footnotetext{
"Corresponding author.

How to cite this paper: Hassan, F.M., Salman, J.M., Dou Abul, A.A. and Naji, A.S. (2016) Polycyclic Aromatic Hydrocarbon (PAHs) Concentrations in Some Aquatic Macrophytes in Hilla River, Iraq. Journal of Environmental Protection, 7, $198-211$. http://dx.doi.org/10.4236/jep.2016.72018
} 


\section{Introduction}

The race to build factories in different countries of the world increases environmental degradation caused by various pollutants and one of these pollutants is polycyclic aromatic hydrocarbons (PAHs). PAHs are a class of organic compounds consisting of two or more aromatic rings [1]. Based on their formation, PAHs can be pyrogenic, petrogenic, diagenic or biogenic [2] [3]. Pyrogenic PAHs are derived from the incomplete combustion of various fuels, oil and gas, garbage, or other organic substances like tobacco or charbroiled meat [4].

PAHs exist in the environment and are distributed in both aquatic and terrestrial environments. Due to their properties such as low aqueous solubility and hydrophobic nature, PAHs are most likely found bound in soils and sediments, and accumulated in the food chain, due to affinity to fatty tissues of organisms [5]. PAHs constituents of non-aqueous phase liquids, make them largely unavailable to microorganisms [6]. Of particular interest in the environment is the acute lethal and sub-lethal toxic effect in freshwater organisms at very low aqueous concentrations [7].

The uptake of large molecules by plant cells is difficult depending on the structure of the cell wall system, especially when they are lipophilic [8].

Prasad et al. [9] revealed that aquatic plants have the ability to uptake bioavailable compounds through their thin cuticle. Rooted aquatic plants have a bioavailabitiy role [10] [11].

Most plant species are sensitive to PAHs to some degree since PAHs can limit primary productivity and constrain total biological activity in an ecosystem [10]. Accumulation of PAHs by plants represents an entry point of hazardous compounds into the food web, initiating a biomagnification process [12] [13]. Plants can be used as a guard species for PAH contamination detection in the environment [14]. Further, a rapid assessment of negative impacts of PAHs can be detected by using plants as a bioindicator [15] [16]. As an added benefit, bioindicators reveal a great deal about the underlying mechanisms of toxicity [17] [18]. Plants that can tolerate contaminated sites can generate a large biomass to remediate PAHs [8] [19].

In aquatic systems, assimilation of contaminants by plants is rapid and efficient, even from sediment, and because of higher affinity of organics to plant tissues than the aqueous phase, the BCFs can be very high [20]. Plants can also assimilate organics following aerial deposition on the leaves [21], thus the contaminants received in this manner can be highly toxic, and represent an important entry point of organic compounds into the food chain. Plants grown in areas with high PAH loads in the soil or air have high bioconcentrations of PAHs [12], because PAHs are lipophilic and tend to accumulate in plants, especially in membrane bilayers [20] [21].

The present study dealt with the fate of PAHs in some macrophytes, in addition to their concentrations and origin of some PAHs in macrophytes.

\section{Material and Methods}

\subsection{Study Area}

Six sites were selected along Al-Hilla River (Figure 1) as described in Hassan et al. [22] and Salman et al. [5]. Table 1 illustrates global positioning system (GPS) locations.

The studied macrophytes (Phragmites australis, Potamogeton pectinatus, Potamogeton perfoliatus and Ceratophyllum demersum) were observed at all sites. Samples were collected between March 2010 and February 2011.

\begin{tabular}{ccc|}
\multicolumn{3}{l}{ Table 1. Coordinates for studied sites. } \\
\hline Site & Longitude (East) & Latitude (North) \\
\hline 1 & $44^{\circ} 18^{\prime} 16.62^{\prime \prime}$ & $32^{\circ} 40^{\prime} 52.32^{\prime \prime}$ \\
2 & $44^{\circ} 16^{\prime} 40.33^{\prime \prime}$ & $32^{\circ} 46^{\prime} 26.40^{\prime \prime}$ \\
3 & $44^{\circ} 23^{\prime} 19.92^{\prime \prime}$ & $32^{\circ} 33^{\prime} 13.57^{\prime \prime}$ \\
4 & $44^{\circ} 26^{\prime} 22.85^{\prime \prime}$ & $32^{\circ} 28^{\prime} 59.81^{\prime \prime}$ \\
5 & $44^{\circ} 29^{\prime} 16.15^{\prime \prime}$ & $32^{\circ} 25^{\prime} 18.51^{\prime \prime}$ \\
6 & $44^{\circ} 39^{\prime} 10.41^{\prime \prime}$ & $32^{\circ} 22^{\prime} 17.77^{\prime \prime}$ \\
\hline
\end{tabular}




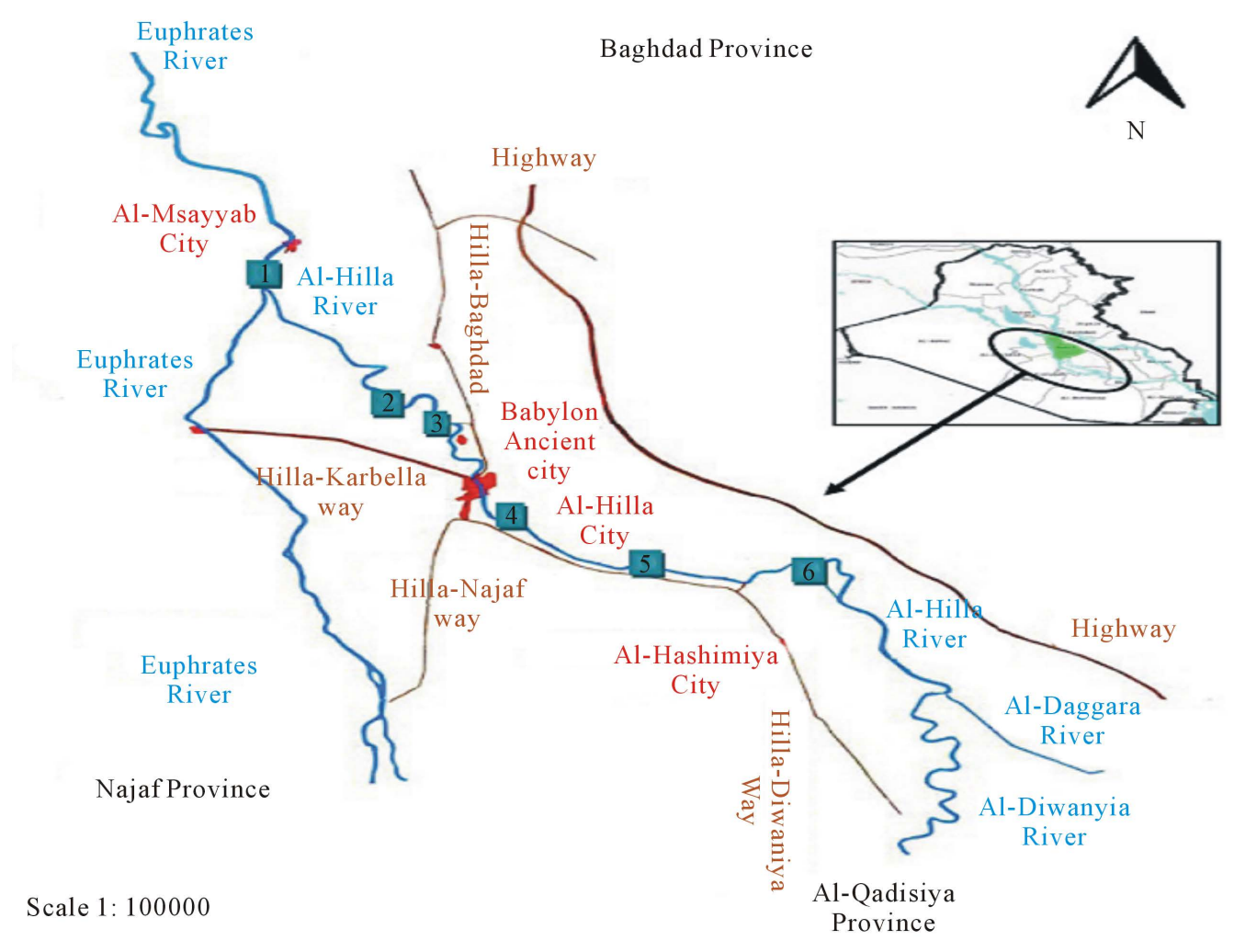

Figure 1. Map of the study area.

Data pertaining to the physicochemical characterization and PAH concentrations in water and sediment of AlHilla River was taken from Hassan et al. [22].

\subsection{Macrophytes Sampling}

Macrophyte samples were sequentially washed with river water, tap water, and distilled water, dried at $15^{\circ} \mathrm{C}$ and wrapped in aluminum. Fresh samples were also wrapped, labeled and frozen at $-20^{\circ} \mathrm{C}$ for subsequent lipid content determination [23].

\subsection{Extraction of PAHs}

Dried specimens were sieved (63 mesh sieve) and $10 \mathrm{~g}$ of plant material were well mixed in a metal blender with $50 \mathrm{ml}$ acetone for 5 minutes. The solution was left overnight in a dark and cold location. After shaking for one hour, the solution was separated and the extract placed in dark glass containers. This step was repeated three times [24]. The solution was centrifuged at 2500 RPM for 5 minutes. Then the supernatant solution was transferred to a flask with $50 \mathrm{ml}$ Hexan and $100 \mathrm{ml}$ deionized water. Upon separation, the upper layer was collected and $50 \mathrm{ml} \mathrm{KOH} \mathrm{(20} \mathrm{ml} \mathrm{aqueous} \mathrm{solution} \mathrm{in} \mathrm{ethanol)} \mathrm{was} \mathrm{added.} \mathrm{The} \mathrm{solution} \mathrm{was} \mathrm{reduced} \mathrm{to} 10 \mathrm{ml}$ by rotary evaporator and transferred to a cleanup process [25].

\subsection{Clean-Up Process}

Because the extract contains complex components, the clean-up procedure was undertaken by column chromatography using $25 \mathrm{~cm}$ of deactivated silica gel (60 - 120 mesh) packed in a glass column (250 mm $\times 15 \mathrm{~mm}$ internal diameter) and Tetrachloromethane for six hours, followed by heat activation at $250^{\circ} \mathrm{C}$ for 12 hours and then cooled and deactivated with water (10\%). After deactivation, the solution was stored in an air-tight dark glass and used within 72 hours. The column was pre-eluted with $10 \mathrm{ml}$ Hexane and the extract was passed through the column and eluted with $50 \mathrm{ml}$ Benzene to separate all PAH compounds [23] [26]. 


\subsection{Lipid Determination}

In a pre-weighted round flask, add $10 \mathrm{~g}$ wet homogenate tissue and $50 \mathrm{~g}$ anhydrous sodium to $250 \mathrm{ml}$ of acetone. Allow to evaporate to dryness and re-weigh. The difference between these weights of flask referrers to lipid content [24].

\subsection{Blank}

Laboratory reagents and glassware were analyzed with each sample to check if any interference that may have been introduced during the extraction and analytical procedure [27] [28].

\subsection{Analysis of PAHs}

Both hexane and benzene were evaporated to dryness by rotary evaporator and the residue was dissolved with 1 $\mathrm{ml}$ (90:10) Acetonitrile:Methanol. The extract was stored at $-20^{\circ} \mathrm{C}$ until analysis by high performance liquid chromatography.

\subsection{Standard Solution}

A standard solution of sixteen US EPA priority PAHs was obtained from Sigma-Aldrich Corporation in order to compare the retention times and spectra of compounds in the standard with those in the sample. The standard calibration contains the following compounds:

Naphthalene, Acenaphthylene, Acenaphthene, Fluorene, Phenanthrene [PHE], Anthracene [ANT], Fluoranthene, Pyrene, Benzo(a) anthracene [B (a)A], Chrysene [Chry], Benzo(b)Fluoranthene [B(b)F], Benzo(k) Fluoranthene $[\mathrm{B}(\mathrm{k}) \mathrm{F}]$, Benzo(a) pyrene $[\mathrm{B}(\mathrm{a}) \mathrm{P}]$, Dibenzo $(\mathrm{a}, \mathrm{h})$ anthracene $[\mathrm{D}(\mathrm{a}, \mathrm{b}) \mathrm{A}]$, Benzo (ghi) Perylene [B(ghi) $\mathrm{P}]$ and Indeno(1,2,3-cd) pyrene [Ind].

\subsection{Bioconcentration Factor (BCF) and Biosediment Accumulation Factor (BSAF)}

$\mathrm{BCF}(\mathrm{l} / \mathrm{g})=\mathrm{PAH}$ concentration in specimen/PAH concentration in water.

BSAF $=$ PAH concentration in specimen $(\mu \mathrm{g} / \mathrm{g}) / \mathrm{PAH}$ concentration in sediment $(\mu \mathrm{g} / \mathrm{g}) \times$ Total Organic Carbon $(\mu \mathrm{g} / \mathrm{g}) / \mathrm{Lipid}$ content $(\mu \mathrm{g} / \mathrm{g})$.

\subsection{Toxicity of Carcinogenic PAHs}

Seven carcinogenic PAHs (c-PAHs) were selected according to EPA (1993): B (a) P, B (a) A, B (b) F, B (k)F, Chry, DbA and Ind (Table 2). The toxicity equivalency factor (TEF) was calculated (see below) to assess the risks of a mixture with a related compound Method B cleanup level (EPA, 1993) relative to B (a) P).

$\mathrm{TEF}=$ Concentration of c-PAH $\times$ equivalent related compound.

Total Toxicity Equivalence Concentration $($ TTEC) $=\Sigma$ TEF .

It must not exceed the method by cleanup level for B (a) P (0.137 $\mu \mathrm{g} / \mathrm{g})$ [29] [30].

The average concentration for each PAH compound and maximum mean for it were compared with standard criteria in Table 2.

Table 2. Standard criteria for Equivalent c-PAHs [31].

\begin{tabular}{cc}
\hline Compounds & Equivalent \\
\hline B (a) P & 1 \\
B (a) A & 0.1 \\
B (b) F & 0.1 \\
B (k) F & 0.1 \\
Chry & 0.01 \\
DbA & 0.1 \\
IND & 0.1 \\
\hline
\end{tabular}




\subsection{PAH Origin}

The PAH origin was assessed according to ratios (Phe/Ant, Chry/BaA, Flu/Pyr, Flu/(Flu + Pyr) and low molecular weight/high molecular weight [32] [33].

\subsection{Statistical Analysis}

The results were analyzed statistically by SPSS (ANOVA, Mean and Standard Deviation) and Canoco for Windows 4.5 (CCA) for the relationships among all tests in the current study.

\section{Results and Discussion}

Many studies have considered lower and higher plants as bioindicators and biomonitors [34] [35], and also the usage of aquatic plants in wastewater treatment, detoxification and phytoremediation [36]-[38].

The results of quality and quantity of PAH compounds in selected aquatic plants are shown in Tables 3-6. The characteristic values of selected molecular ratios for pyrogenic and petrogenic origins of PAHs in the studied plants are Tables 7-10.

The mean range of PAHs in macrophytes were as follows: P. australis $0.425-299.424 \mu \mathrm{g} / \mathrm{g} \mathrm{DW}, P$. perfoliatus 0.354 - $235.84 \mu \mathrm{g} / \mathrm{g}$ DW, C. demersum 0.996 - $162.942 \mu \mathrm{g} / \mathrm{g}$ DW, and P. Pectinatus 0.383 - $99.87 \mu \mathrm{g} / \mathrm{g}$ DW (Figure 2). The variation between aquatic plants may be due to the ability of $P$. australis to absorb the pollutants from sediment and water [39] [40], and its high growth rate and luxury accumulation of major nutrients in stems, roots, and rhizomes [41]-[43]. Other studied macrophytes exhibit lower values because these plants are non-rooted submerged macrophytes, which depend on compound availability in the water column [44] [45].
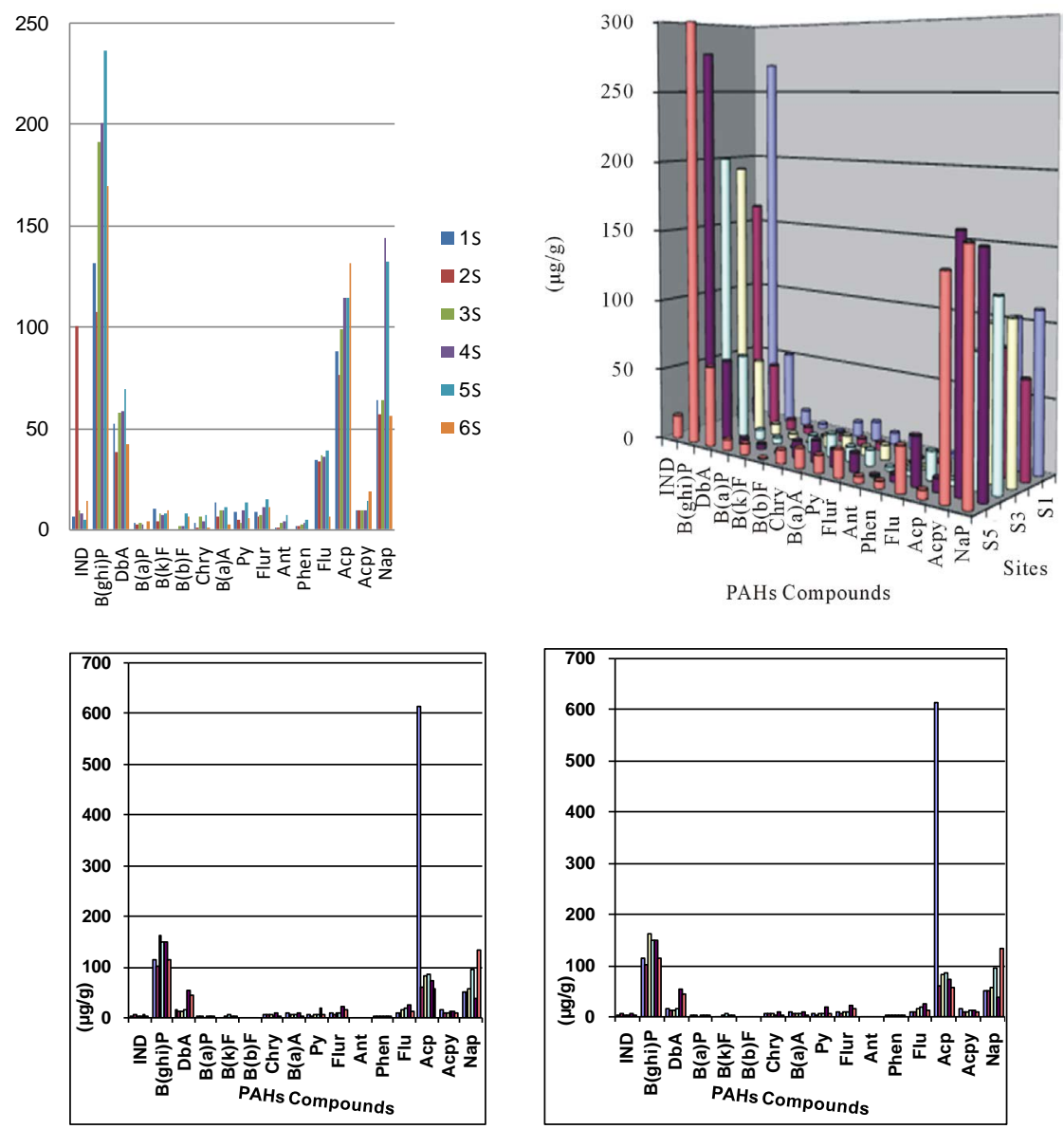

Figure 2. PAHs concentrations in the studied sites for $P$. perfoliatus, $P$. australis, C. demersum and $P$. pectinatus. 
Table 3. Values of PAHs compounds in P. pectinatus during March 2010-February 2011 (S = Speing, S = Summer, A = Autumn, $\mathrm{W}=$ Winter).

\begin{tabular}{|c|c|c|c|c|c|c|c|c|c|c|c|c|c|c|c|c|c|}
\hline \multirow{2}{*}{ Station } & \multirow{2}{*}{ Season } & \multicolumn{16}{|c|}{ PAHs Compounds $(\mu \mathrm{g} / \mathrm{g})$} \\
\hline & & Nap & Aсру & Acp & Flu & Phen & Ant & Flur & PY & $\mathrm{B}(\mathrm{a}) \mathrm{A}$ & Chry & $\mathrm{B}(\mathrm{b}) \mathrm{F}$ & $\mathrm{B}(\mathrm{k}) \mathrm{F}$ & $\mathrm{B}(\mathrm{a}) \mathrm{P}$ & $\mathrm{D}(\mathrm{b}) \mathrm{A}$ & B(ghi)P & IND \\
\hline \multirow{6}{*}{1} & $\mathrm{~S}$ & 73.56 & 71.26 & ND & ND & 5.06 & 0.76 & ND & ND & 10.55 & 7.23 & 1.76 & 7.55 & 3.72 & 52.91 & 84.56 & 2.45 \\
\hline & $\mathrm{S}$ & 14.75 & 16.71 & 1.03 & 0.00 & ND & ND & 0.58 & 1.04 & 1.67 & ND & ND & 1.82 & 0.16 & 10.36 & 22.47 & 0.96 \\
\hline & A & 79.93 & 78.99 & 6.07 & 30.80 & ND & $\mathrm{ND}$ & 2.75 & ND & ND & 3.58 & 3.33 & 7.90 & 3.00 & 50.44 & 84.03 & 2.58 \\
\hline & W & ND & ND & ND & 1.15 & 0.98 & 1.01 & 7.30 & 3.98 & 7.81 & 7.69 & ND & 11.31 & 3.05 & ND & 82.48 & 2.39 \\
\hline & Mean & 59.95 & 41.74 & 3.69 & 7.99 & 3.85 & 0.44 & 3.61 & 2.01 & 5.01 & 4.62 & 1.27 & 7.14 & 2.48 & 28.43 & 68.38 & 2.09 \\
\hline & \pm S.D. & 30.34 & 39.72 & 3.74 & 15.22 & 2.91 & 0.51 & 2.80 & 1.80 & 4.99 & 3.59 & 1.60 & 3.93 & 1.58 & 27.19 & 30.62 & 0.76 \\
\hline \multirow{6}{*}{2} & $\mathrm{~S}$ & 62.83 & 98.01 & 5.35 & 7.45 & 2.06 & ND & ND & 5.27 & 13.35 & 4.32 & 1.16 & ND & 3.96 & 88.13 & 62.49 & 1.34 \\
\hline & $\mathrm{S}$ & 12.55 & ND & ND & ND & 0.48 & 0.00 & ND & ND & 2.01 & 0.54 & ND & 0.00 & 0.48 & 0.00 & 25.01 & 0.55 \\
\hline & A & 67.29 & 97.28 & ND & ND & 2.34 & 0.97 & ND & ND & 11.97 & 3.60 & ND & 4.44 & 2.00 & 81.14 & 84.27 & 1.55 \\
\hline & W & 70.67 & 91.67 & 10.11 & 1.25 & ND & ND & 8.07 & ND & ND & ND & 1.31 & 6.97 & ND & 84.17 & 79.66 & 2.79 \\
\hline & Mean & 53.33 & 80.10 & 7.73 & 2.17 & 1.66 & 0.38 & 3.82 & 2.52 & 6.83 & 2.11 & 0.68 & 2.85 & 1.61 & 63.36 & 62.86 & 1.56 \\
\hline & \pm S.D. & 23.37 & 31.23 & 5.20 & 3.56 & 0.82 & 0.47 & 3.70 & 2.91 & 6.80 & 2.16 & 0.65 & 3.45 & 1.78 & 42.33 & 26.91 & 0.92 \\
\hline \multirow{6}{*}{3} & $\mathrm{~S}$ & 54.22 & 59.78 & 3.33 & ND & ND & 0.93 & 12.39 & 7.69 & 6.34 & ND & 1.22 & 3.98 & 1.89 & 47.39 & 81.66 & 1.03 \\
\hline & $\mathrm{S}$ & 12.59 & ND & ND & ND & 0.46 & 0.00 & 0.00 & 2.89 & 0.26 & 0.00 & 0.64 & 0.38 & ND & 0.00 & ND & 0.73 \\
\hline & A & 66.33 & ND & ND & ND & 1.38 & 1.91 & 13.55 & 0.00 & 7.78 & 7.25 & 2.97 & 2.88 & ND & 47.78 & ND & 1.24 \\
\hline & W & 57.24 & ND & ND & ND & 0.11 & 0.05 & 10.93 & 9.11 & 12.98 & 15.67 & 1.62 & 3.22 & $\mathrm{ND}$ & 45.35 & 86.59 & 1.14 \\
\hline & Mean & 47.59 & 46.13 & 1.70 & 7.81 & 0.49 & 0.72 & 9.22 & 4.92 & 6.84 & 5.73 & 1.61 & 2.62 & 0.62 & 35.13 & 42.06 & 1.03 \\
\hline & \pm S.D. & 31.45 & 39.18 & 1.46 & 6.82 & 0.62 & 0.89 & 6.23 & 3.60 & 4.22 & 7.45 & 0.99 & 1.55 & 0.89 & 23.44 & 48.60 & 0.22 \\
\hline & $\mathrm{S}$ & 82.23 & 36.81 & ND & ND & 0.96 & 1.05 & 4.51 & ND & 9.62 & 5.61 & 2.32 & 4.17 & ND & 32.41 & 160.61 & ND \\
\hline & $\mathrm{S}$ & 23.36 & 11.42 & ND & 1.34 & 0.25 & ND & 1.59 & 0.48 & $\mathrm{ND}$ & 0.89 & 0.14 & 1.96 & 0.83 & ND & 77.34 & 0.92 \\
\hline & A & 44.16 & 42.82 & 10.58 & 8.40 & 1.77 & $\mathrm{ND}$ & 3.56 & 5.56 & 6.64 & 8.96 & ND & 2.32 & 2.34 & 5.81 & 105.31 & 1.06 \\
\hline & W & 29.97 & 77.25 & 13.92 & 8.87 & 1.48 & 0.62 & 6.84 & 5.80 & 8.40 & 5.20 & 1.36 & 1.71 & ND & 33.92 & ND & 1.59 \\
\hline & Mean & 44.93 & 42.07 & 6.12 & 4.65 & 1.11 & 0.42 & 4.124 & 2.96 & 6.17 & 5.17 & 0.96 & 2.54 & 0.79 & 18.03 & 85.81 & 0.89 \\
\hline & \pm S.D. & 26.33 & 27.11 & 7.20 & 4.63 & 0.66 & 0.51 & 2.17 & 3.14 & 4.28 & 3.31 & 1.09 & 1.11 & 1.10 & 17.64 & 66.85 & 0.66 \\
\hline \multirow{6}{*}{5} & $\mathrm{~S}$ & 147.48 & 86.69 & ND & 2.78 & 3.59 & $\mathrm{ND}$ & 8.50 & 4.08 & ND & 6.20 & 11.32 & 10.81 & 0.00 & ND & 170.38 & 9.45 \\
\hline & $\mathrm{S}$ & 32.13 & 21.57 & ND & 0.59 & 0.97 & ND & 1.26 & 0.20 & ND & 1.33 & 0.01 & 0.76 & 1.88 & ND & 33.48 & 0.25 \\
\hline & A & 121.01 & 98.25 & 7.45 & 3.58 & 1.13 & 1.59 & 9.48 & ND & 13.36 & 4.25 & 10.06 & 12.15 & 9.48 & ND & 87.71 & 9.11 \\
\hline & W & 98.90 & 90.97 & ND & 4.73 & 0.07 & 0.07 & 8.39 & 5.10 & 15.83 & 4.37 & 24.32 & 12.85 & $\mathrm{ND}$ & 83.58 & 88.53 & ND \\
\hline & Mean & 99.88 & 74.37 & 1.86 & 2.92 & 1.44 & 0.42 & 6.91 & 2.34 & 10.68 & 4.03 & 11.43 & 9.14 & 2.83 & 37.03 & 95.02 & 4.70 \\
\hline & $\pm \mathrm{SD}$ & 49.34 & 35.52 & 1.74 & 2.07 & 1.50 & 0.78 & 3.79 & 2.62 & 7.20 & 2.01 & 9.97 & 5.65 & 4.51 & 43.46 & 56.45 & 5.28 \\
\hline & $\mathrm{S}$ & 39.10 & 75.35 & 5.43 & 12.23 & ND & 2.36 & 6.62 & 6.54 & 4.45 & 4.91 & 1.04 & ND & ND & 25.08 & 184.78 & 0.33 \\
\hline & $\mathrm{S}$ & 12.37 & 18.55 & 3.48 & 2.26 & 0.42 & ND & ND & 1.25 & ND & 1.32 & ND & 1.25 & 0.78 & ND & 22.98 & ND \\
\hline & A & 28.71 & 57.60 & ND & 13.56 & 1.28 & 2.66 & 4.57 & ND & 5.55 & 3.78 & ND & 4.77 & 0.99 & 19.59 & 108.70 & ND \\
\hline & W & 11.63 & 39.99 & 8.18 & 18.46 & 2.99 & 1.20 & 6.76 & 6.80 & 5.13 & 4.93 & 0.77 & 7.57 & $\mathrm{ND}$ & 63.63 & ND & 1.15 \\
\hline & Mean & 22.95 & 47.87 & 4.27 & 11.63 & 1.17 & 1.55 & 4.49 & 3.65 & 3.78 & 3.73 & 0.45 & 3.40 & 0.44 & 27.07 & 79.11 & 0.36 \\
\hline & \pm S.D. & 13.34 & 24.30 & 3.43 & 6.79 & 1.32 & 1.21 & 3.15 & 3.52 & 2.56 & 1.69 & 0.53 & 3.43 & 0.51 & 26.63 & 84.55 & 0.54 \\
\hline
\end{tabular}


Table 4. Values of PAHs compounds in P. australis during March 2010-February 2011 (S = Spring, S = Summer, A = Autumn., $\mathrm{W}=$ Winter).

\begin{tabular}{|c|c|c|c|c|c|c|c|c|c|c|c|c|c|c|c|c|c|}
\hline \multirow{2}{*}{ tation } & \multirow{2}{*}{ Season } & \multicolumn{16}{|c|}{ PAHs Compounds ( $\mu \mathrm{g} / \mathrm{g})$} \\
\hline & & Nap & Aсру & Acp & Flu & Phen & Ant & Flur & PY & $\mathrm{B}(\mathrm{a}) \mathrm{A}$ & Chry & $\mathrm{B}(\mathrm{b}) \mathrm{F}$ & $\mathrm{B}(\mathrm{k}) \mathrm{F}$ & $\mathrm{B}(\mathrm{a}) \mathrm{P}$ & $\mathrm{DbA}$ & B(ghi)P & IND \\
\hline \multirow{6}{*}{1} & $\mathrm{~S}$ & 108.53 & 107.13 & 36.19 & ND & 2.26 & 1.56 & ND & 13.23 & 33.51 & 23.26 & ND & ND & 13.06 & ND & 469.30 & 11.34 \\
\hline & $\mathrm{S}$ & 42.54 & 97.45 & ND & ND & 6.01 & 1.37 & 7.50 & ND & 3.27 & 9.52 & 1.64 & 2.34 & 10.24 & 41.51 & 154.29 & 0.44 \\
\hline & A & 240.34 & 123.52 & 15.13 & ND & 1.33 & 1.51 & 13.98 & 10.71 & 3.18 & ND & 1.75 & 6.70 & 16.85 & 73.59 & 186.82 & 21.99 \\
\hline & W & 46.52 & 82.51 & 1.92 & 13.93 & 0.95 & ND & 16.73 & 9.70 & 13.65 & 9.03 & ND & 4.70 & 2.52 & 88.71 & 267.85 & 14.42 \\
\hline & Mean & 109.48 & 102.65 & 13.31 & 3.48 & 2.64 & 1.11 & 9.55 & 8.40 & 13.40 & 10.45 & 0.85 & 3.44 & 10.67 & 50.95 & 269.56 & 12.05 \\
\hline & \pm S.D. & 29.32 & 17.20 & 16.67 & 8.04 & 2.31 & 0.74 & 7.45 & 5.79 & 14.27 & 9.59 & 0.98 & 2.90 & 6.06 & 39.25 & 141.45 & 8.93 \\
\hline \multirow{7}{*}{2} & $\mathrm{~S}$ & ND & 144.17 & ND & 1.11 & 0.25 & 0.26 & 0.62 & 0.66 & 0.75 & 0.11 & 1.45 & 4.49 & 2.80 & 1.95 & 105.83 & ND \\
\hline & $S$ & 22.58 & 45.02 & ND & ND & 4.15 & 0.92 & 3.84 & ND & 3.43 & 10.35 & 1.70 & 2.37 & 10.58 & 43.97 & 121.09 & 0.37 \\
\hline & A & 200.42 & 71.09 & 13.95 & ND & 1.14 & 1.37 & 10.33 & 6.30 & 4.00 & ND & 1.60 & 6.13 & 14.82 & 71.12 & 178.52 & 22.70 \\
\hline & W & 45.93 & 81.46 & 13.71 & 13.02 & 0.49 & ND & 10.38 & 14.11 & 12.02 & 8.53 & ND & 4.70 & 1.16 & 65.72 & 251.25 & 14.28 \\
\hline & Mean & 67.23 & 85.43 & 6.92 & 3.53 & 1.51 & 0.64 & 6.29 & 5.27 & 5.05 & 4.75 & 1.18 & 4.42 & 7.34 & 45.69 & 164.17 & 9.34 \\
\hline & \pm S.D. & 90.75 & 42.04 & 7.98 & 6.34 & 1.79 & 0.62 & 4.87 & 6.54 & 4.85 & 5.47 & 0.79 & 1.55 & 6.46 & 31.43 & 65.94 & 11.11 \\
\hline & $\mathrm{S}$ & 95.38 & 187.16 & 0.30 & 0.70 & 0.06 & ND & 10.43 & 0.17 & 12.30 & 1.42 & ND & 0.12 & ND & 35.25 & 262.65 & 1.11 \\
\hline & $\mathrm{S}$ & 42.54 & 42.40 & ND & 17.68 & 4.05 & 0.91 & 3.66 & 13.40 & 4.25 & 11.18 & ND & 2.39 & ND & 44.79 & 204.09 & 0.44 \\
\hline & A & 242.34 & 97.30 & ND & ND & 2.08 & 5.89 & 10.69 & 6.74 & 12.13 & 10.68 & 1.70 & 6.70 & 21.60 & 79.33 & 203.42 & 21.99 \\
\hline & W & 56.50 & 90.37 & 13.71 & 15.23 & 1.05 & ND & 17.09 & 5.28 & 15.27 & 10.70 & 0.00 & 4.70 & 9.31 & 47.66 & 101.85 & 19.38 \\
\hline & Mean & 109.19 & 104.31 & 3.50 & 8.40 & 1.81 & 1.70 & 10.47 & 6.40 & 10.90 & 8.50 & 0.43 & 3.48 & 7.73 & 51.76 & 193.00 & 10.73 \\
\hline & 土S.D. & 91.53 & 60.38 & 6.80 & 9.35 & 1.70 & 2.82 & 5.48 & 5.45 & 4.72 & 4.72 & 0.85 & 2.84 & 10.23 & 19.13 & 66.81 & 11.54 \\
\hline \multirow{6}{*}{4} & $\mathrm{~S}$ & 115.34 & 29.87 & 0.27 & 2.00 & 0.62 & ND & 0.80 & 0.61 & 10.43 & 9.75 & ND & 0.70 & ND & 43.46 & 179.65 & 8.19 \\
\hline & $\mathrm{S}$ & 46.53 & 94.83 & ND & 43.68 & 7.78 & 4.06 & 25.57 & 17.81 & 12.38 & 10.35 & ND & 2.68 & ND & 47.26 & 170.89 & 0.51 \\
\hline & A & 222.38 & 97.04 & ND & ND & 1.14 & 6.11 & 7.04 & 6.65 & 20.26 & 10.68 & 1.75 & 3.83 & 20.92 & 71.12 & 120.42 & 21.28 \\
\hline & W & 117.78 & 133.89 & 16.07 & 32.52 & 4.77 & ND & 10.75 & 15.00 & 20.15 & 16.86 & 2.11 & 10.45 & 7.95 & 72.29 & 334.25 & ND \\
\hline & Mean & 125.51 & 88.91 & 4.08 & 19.55 & 3.58 & 2.54 & 11.04 & 10.02 & 15.81 & 11.91 & 0.97 & 4.42 & 7.22 & 58.53 & 201.30 & 7.50 \\
\hline & \pm S.D. & 72.53 & 43.24 & 7.99 & 21.91 & 3.35 & 3.05 & 10.52 & 7.86 & 5.14 & 3.32 & 1.12 & 4.22 & 9.87 & 15.29 & 92.39 & 9.92 \\
\hline & $\mathrm{S}$ & 155.26 & 213.37 & 7.77 & 15.01 & 2.48 & ND & 11.76 & 18.27 & 18.57 & 18.07 & ND & 0.73 & ND & 59.87 & 262.65 & 29.42 \\
\hline & $\mathrm{S}$ & 86.45 & 147.26 & ND & 46.28 & 8.06 & 4.38 & 8.13 & 3.90 & 13.19 & 11.18 & ND & 2.39 & ND & 48.08 & 253.89 & 1.22 \\
\hline & A & 103.98 & 145.60 & 1.35 & ND & 1.69 & 0.51 & 17.55 & 13.71 & 1.39 & ND & 1.16 & 0.86 & 6.13 & 42.26 & 223.15 & 11.61 \\
\hline & W & 285.43 & 160.10 & 27.87 & 71.53 & 8.50 & ND & 14.40 & 13.24 & 28.28 & 18.52 & 2.32 & 11.02 & 13.38 & 80.50 & 367.45 & ND \\
\hline & Mean & 157.778 & 166.58 & 9.25 & 33.20 & 5.18 & 1.22 & 12.96 & 12.28 & 15.36 & 11.94 & 0.87 & 3.75 & 4.88 & 57.68 & 276.78 & 10.56 \\
\hline & $\pm \mathrm{SD}$ & 89.96 & 31.85 & 12.86 & 32.00 & 3.59 & 2.11 & 3.99 & 6.02 & 11.21 & 8.64 & 1.10 & 4.90 & 6.36 & 16.88 & 62.77 & 13.60 \\
\hline & $\mathrm{S}$ & 170.17 & 144.44 & ND & 10.74 & 3.96 & 4.16 & 23.06 & ND & 22.22 & 6.00 & ND & 4.08 & ND & 27.74 & 364.64 & 0.47 \\
\hline & $\mathrm{S}$ & 88.45 & 149.88 & ND & 44.98 & 7.87 & 4.11 & 6.67 & 8.26 & 9.94 & 12.02 & ND & 2.71 & ND & 56.29 & 212.39 & 1.93 \\
\hline & A & 142.54 & 123.26 & ND & ND & 0.03 & 10.62 & 23.39 & 12.24 & 24.00 & 4.02 & 0.70 & 15.32 & 18.89 & 62.91 & 286.42 & 37.91 \\
\hline & W & 245.51 & 157.48 & 24.33 & 67.62 & 8.40 & ND & 24.03 & 28.68 & ND & 16.86 & 2.16 & 9.30 & 12.02 & 78.86 & 334.25 & 25.33 \\
\hline & Mean & 161.67 & 143.77 & 6.08 & 30.84 & 5.06 & 4.72 & 19.29 & 12.29 & 14.04 & 9.72 & 0.72 & 7.87 & 7.73 & 56.45 & 299.42 & 16.410 \\
\hline & 土S.D. & 65.39 & 14.68 & 12.16 & 35.86 & 3.89 & 4.38 & 8.42 & 13.61 & 11.25 & 5.84 & 1.01 & 5.73 & 9.35 & 21.35 & 66.35 & 18.30 \\
\hline
\end{tabular}


Table 5. Values of PAHs compounds in C. demersum during March 2010-February 2011 (S = spring, $\mathrm{S}=$ summer, $\mathrm{A}=$ autumn, $\mathrm{W}=$ winter).

\begin{tabular}{|c|c|c|c|c|c|c|c|c|c|c|c|c|c|c|c|c|c|}
\hline \multirow{2}{*}{ Station } & \multirow{2}{*}{ Season } & \multicolumn{16}{|c|}{ PAHs Compounds $(\mu \mathrm{g} / \mathrm{g})$} \\
\hline & & Nap & Aсру & Acp & Flu & Phen & Ant & Flur & PY & $\mathrm{B}(\mathrm{a}) \mathrm{A}$ & Chry & $\mathrm{B}(\mathrm{b}) \mathrm{F}$ & $\mathrm{B}(\mathrm{k}) \mathrm{F}$ & $\mathrm{B}(\mathrm{a}) \mathrm{P}$ & $\mathrm{D}(\mathrm{b}) \mathrm{A}$ & B(ghi)P & IND \\
\hline \multirow{6}{*}{ 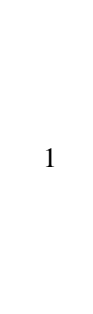 } & $\mathrm{S}$ & 66.11 & ND & ND & ND & 2.14 & ND & ND & 13.28 & 10.27 & 6.87 & 0.81 & 0.35 & 5.38 & 24.04 & 157.07 & 1.34 \\
\hline & $\mathrm{S}$ & 21.89 & ND & ND & 27.83 & 4.21 & ND & ND & 0.83 & 7.91 & 10.18 & 0.14 & 1.20 & ND & 12.37 & 78.16 & 0.43 \\
\hline & A & 62.60 & 26.78 & 83.55 & 15.69 & ND & ND & 19.08 & 9.38 & ND & $\mathrm{ND}$ & ND & 3.93 & 9.68 & 15.17 & 113.16 & 7.81 \\
\hline & W & 54.27 & 28.61 & ND & ND & 5.57 & 1.30 & 19.57 & 8.31 & 11.99 & 13.69 & 5.03 & 5.12 & ND & 19.84 & 113.42 & 4.65 \\
\hline & Mean & 51.22 & 16.16 & 614.94 & 10.88 & 5.55 & 1.53 & 9.66 & 7.95 & 11.82 & 7.69 & 1.49 & 2.65 & 3.76 & 17.85 & 115.45 & 3.56 \\
\hline & \pm S.D. & 20.17 & 13.86 & 41.31 & 13.50 & 3.44 & 1.56 & 11.15 & 5.20 & 3.90 & 5.83 & 2.38 & 2.24 & 4.68 & 5.14 & 32.31 & 3.36 \\
\hline \multirow{6}{*}{2} & $\mathrm{~S}$ & 62.12 & ND & 64.24 & ND & 1.96 & 0.51 & $\mathrm{ND}$ & 4.45 & 2.14 & 7.71 & 0.76 & 0.06 & 4.70 & 15.83 & 130.60 & 15.83 \\
\hline & $\mathrm{S}$ & 17.90 & 6.28 & ND & 26.53 & 3.28 & 3.63 & ND & 0.28 & 7.66 & 11.01 & 0.13 & 1.23 & ND & 12.45 & 67.67 & 1.14 \\
\hline & A & 70.61 & 24.42 & 90.35 & 13.09 & 9.33 & ND & 11.35 & 4.94 & 16.29 & ND & ND & 3.35 & 9.00 & 8.60 & 110.54 & 7.10 \\
\hline & W & 52.27 & 8.26 & 88.47 & ND & 4.62 & 0.85 & 15.92 & 7.89 & 8.74 & 12.03 & 5.13 & 5.70 & ND & 20.66 & 105.56 & 4.44 \\
\hline & Mean & 50.72 & 9.74 & 60.77 & 9.90 & 4.80 & 1.25 & 6.82 & 4.39 & 8.71 & 7.69 & 1.51 & 2.59 & 3.42 & 14.38 & 103.59 & 7.13 \\
\hline & \pm S.D. & 23.13 & 10.40 & 42.21 & 12.68 & 3.21 & 1.62 & 8.08 & 3.13 & 5.82 & 5.44 & 2.44 & 2.48 & 4.32 & 5.12 & 26.27 & 6.29 \\
\hline \multirow{6}{*}{3} & $\mathrm{~S}$ & 67.31 & 11.70 & 97.44 & ND & 2.21 & 1.12 & 14.61 & 7.49 & ND & 7.04 & 0.81 & 0.64 & ND & 32.25 & 156.81 & 3.46 \\
\hline & $\mathrm{S}$ & ND & ND & ND & 30.43 & 4.30 & 3.77 & ND & 0.28 & 7.10 & 11.85 & 0.19 & 1.29 & ND & 13.19 & 130.59 & 1.14 \\
\hline & A & ND & ND & 113.35 & 13.22 & 9.61 & ND & 10.98 & 9.20 & 16.37 & $\mathrm{ND}$ & 2.66 & 3.93 & 10.36 & 9.42 & 136.75 & 7.17 \\
\hline & W & 77.55 & 26.87 & 121.70 & 26.03 & 0.88 & 0.70 & 15.00 & 10.42 & 6.92 & 7.97 & 0.73 & 9.14 & ND & ND & 227.62 & 8.63 \\
\hline & Mean & 59.82 & 9.64 & 83.12 & 17.42 & 4.25 & 1.40 & 10.15 & 6.85 & 7.60 & 6.71 & 1.10 & 3.75 & 2.59 & 13.71 & 162.94 & 5.10 \\
\hline & \pm S.D. & 24.33 & 12.74 & 56.32 & 13.71 & 3.83 & 1.64 & 7.00 & 4.54 & 6.71 & 4.93 & 1.07 & 3.86 & 5.18 & 13.54 & 44.54 & 3.41 \\
\hline \multirow{6}{*}{4} & $\mathrm{~S}$ & 100.88 & ND & ND & 43.32 & ND & ND & ND & 11.78 & 3.82 & 5.30 & 0.46 & ND & ND & ND & 151.95 & 1.41 \\
\hline & $\mathrm{S}$ & 51.93 & ND & ND & 4.83 & 3.28 & ND & ND & 3.01 & 7.42 & 8.52 & 0.19 & ND & ND & 14.01 & 51.95 & 0.51 \\
\hline & A & ND & ND & 119.95 & 15.82 & $\mathrm{ND}$ & ND & 22.44 & 10.53 & ND & $\mathrm{ND}$ & 2.82 & 9.67 & 17.15 & 50.46 & 139.37 & 7.17 \\
\hline & W & 117.51 & 24.51 & 130.00 & 13.03 & 0.87 & 0.61 & 24.27 & 9.54 & 6.11 & 8.05 & 0.79 & ND & ND & ND & 253.84 & 10.04 \\
\hline & Mean & 95.19 & 12.91 & 85.41 & 19.25 & 3.67 & 0.95 & 11.68 & 8.71 & 8.55 & 5.47 & 1.06 & 6.13 & 4.29 & 16.12 & 149.27 & 4.78 \\
\hline & \pm S.D. & 29.63 & 11.49 & 59.20 & 16.71 & 4.78 & 1.27 & 13.50 & 3.34 & 5.73 & 3.91 & 1.19 & 3.76 & 8.57 & 23.82 & 82.68 & 4.58 \\
\hline & $\mathrm{S}$ & ND & ND & ND & ND & ND & ND & 21.45 & 24.36 & 15.88 & 16.26 & 1.79 & ND & ND & ND & 172.30 & 3.43 \\
\hline & $\mathrm{S}$ & ND & ND & 33.57 & ND & ND & ND & ND & 10.33 & 8.07 & 13.51 & 0.87 & 4.16 & 5.23 & 46.02 & 78.16 & ND \\
\hline & A & ND & ND & 131.45 & 17.12 & 11.47 & 0.98 & 33.54 & ND & ND & ND & 3.08 & 9.67 & 10.29 & 52.10 & 179.86 & 9.29 \\
\hline & W & 155.88 & ND & ND & 52.06 & $\mathrm{ND}$ & ND & 36.65 & 19.39 & 17.51 & 10.73 & 0.83 & ND & ND & 121.18 & 172.13 & 15.13 \\
\hline & Mean & 38.97 & 14.23 & 74.38 & 25.23 & 4.37 & 1.24 & 22.91 & 19.09 & 10.37 & 10.12 & 1.64 & 5.29 & 3.88 & 54.83 & 150.61 & 6.96 \\
\hline & $\pm \mathrm{SD}$ & 82.58 & 28.46 & 67.90 & 22.09 & 5.02 & 1.88 & 16.61 & 6.18 & 8.04 & 7.11 & 1.05 & 3.71 & 4.93 & 49.97 & 48.43 & 6.66 \\
\hline & $\mathrm{S}$ & ND & ND & 99.04 & 31.17 & 2.46 & 1.10 & 17.67 & 1.94 & 9.29 & 8.13 & ND & ND & 11.40 & 5.90 & 143.80 & 3.30 \\
\hline & $\mathrm{S}$ & 25.65 & 0.91 & ND & ND & 4.26 & ND & ND & 16.12 & 9.89 & 4.69 & 2.03 & ND & ND & ND & 39.09 & 0.73 \\
\hline & A & ND & ND & 98.25 & 14.52 & 9.61 & 0.94 & 211.35 & ND & $\mathrm{ND}$ & ND & 2.71 & 9.10 & 10.36 & 51.28 & 144.61 & 7.88 \\
\hline & W & 185.92 & ND & ND & 4.47 & ND & ND & 22.63 & 0.56 & 1.25 & 2.40 & 0.31 & ND & ND & 129.39 & 139.51 & 8.06 \\
\hline & Mean & 132.94 & 11.51 & 58.90 & 12.54 & 4.27 & 1.00 & 15.41 & 7.06 & 5.11 & 3.80 & 1.42 & 5.65 & 5.44 & 46.64 & 116.75 & 4.99 \\
\hline & \pm S.D. & 93.51 & 22.42 & 48.48 & 13.82 & 3.83 & 0.79 & 10.48 & 7.24 & 5.20 & 3.45 & 1.13 & 4.69 & 6.29 & 59.73 & 51.82 & 3.59 \\
\hline
\end{tabular}


Table 6. Values of PAHs compounds in P. perfoliatus during March 2010-February 2011 (S = spring, $\mathrm{S}=$ summer, $\mathrm{A}=$ autumn, $\mathrm{W}=$ winter).

\begin{tabular}{|c|c|c|c|c|c|c|c|c|c|c|c|c|c|c|c|c|c|}
\hline \multirow{2}{*}{ Station } & \multirow{2}{*}{ Season } & \multicolumn{16}{|c|}{ PAHs Compounds $(\mu \mathrm{g} / \mathrm{g})$} \\
\hline & & Nap & Aсру & Aср & Flu & Phen & Ant & Flur & PY & $\mathrm{B}(\mathrm{a}) \mathrm{A}$ & Chry & $\mathrm{B}(\mathrm{b}) \mathrm{F}$ & $\mathrm{B}(\mathrm{k}) \mathrm{F}$ & $\mathrm{B}(\mathrm{a}) \mathrm{P}$ & $\mathrm{D}(\mathrm{b}) \mathrm{A}$ & $\mathrm{B}$ (ghi)P & IND \\
\hline & $\mathrm{S}$ & 50.42 & ND & 109.02 & 18.21 & 0.10 & 0.14 & 18.91 & ND & 2.67 & 2.66 & 0.24 & 8.32 & ND & 43.37 & 267.84 & 7.21 \\
\hline & $\mathrm{S}$ & 37.88 & 12.02 & 27.85 & 27.41 & ND & ND & ND & 3.05 & 17.17 & 6.21 & ND & 27.27 & 6.21 & 67.31 & 44.91 & 4.16 \\
\hline & A & 57.84 & 27.65 & 101.05 & 33.91 & 4.50 & 2.24 & ND & 14.70 & 23.67 & 5.38 & ND & 2.86 & 6.61 & 73.05 & 45.43 & 9.83 \\
\hline & W & 109.68 & ND & 113.52 & 58.98 & 1.52 & 1.11 & 17.06 & 16.82 & 10.04 & ND & 1.18 & 3.93 & ND & 26.93 & 168.53 & 5.81 \\
\hline & Mean & 63.96 & 9.92 & 87.86 & 34.63 & 1.53 & 0.87 & 8.99 & 8.64 & 13.39 & 3.56 & 0.35 & 10.59 & 3.21 & 52.66 & 131.68 & 6.75 \\
\hline & \pm S.D. & 31.57 & 13.11 & 40.33 & 17.46 & 2.09 & 1.03 & 10.41 & 8.35 & 9.06 & 2.81 & 0.56 & 11.36 & 3.70 & 21.43 & 107.80 & 2.39 \\
\hline & $\mathrm{S}$ & 50.30 & ND & 97.36 & 1.83 & 0.01 & 0.01 & 11.01 & ND & 1.04 & 1.83 & 0.23 & 8.12 & ND & 42.55 & 262.60 & 7.28 \\
\hline & $\mathrm{S}$ & 51.85 & 11.22 & 21.25 & 28.71 & 3.01 & 0.97 & ND & 3.22 & 8.22 & ND & ND & 2.31 & 4.10 & 37.76 & 34.94 & 7.09 \\
\hline & A & 53.85 & 24.12 & 96.15 & 32.61 & 4.22 & 1.87 & ND & 9.05 & 15.54 & 1.21 & ND & 2.80 & 5.46 & 70.59 & 40.19 & 12.75 \\
\hline & W & 69.77 & ND & 90.52 & 71.98 & 0.59 & 0.21 & 12.76 & 5.40 & 0.28 & ND & 0.03 & 1.06 & ND & 2.31 & 89.88 & 12.89 \\
\hline & Mean & 56.44 & 9.11 & 76.32 & 33.78 & 1.96 & 0.77 & 5.94 & 4.42 & 6.27 & 0.76 & 0.06 & 3.57 & 2.39 & 38.30 & 106.90 & 10.00 \\
\hline & \pm S.D. & 9.00 & 11.56 & 36.83 & 28.91 & 1.99 & 0.84 & 6.89 & 3.80 & 7.14 & 0.91 & 0.10 & 3.11 & 2.81 & 28.02 & 106.70 & 3.25 \\
\hline \multirow{6}{*}{3} & $\mathrm{~S}$ & 54.29 & ND & 116.36 & 3.13 & 0.11 & 0.03 & 14.81 & ND & 2.67 & 2.66 & 0.44 & 13.86 & ND & 34.34 & 393.67 & 8.70 \\
\hline & $\mathrm{S}$ & 53.85 & 13.50 & 39.52 & 30.01 & 3.03 & 0.93 & ND & 2.78 & 8.39 & ND & ND & 2.63 & 4.78 & 54.17 & 40.19 & 7.30 \\
\hline & A & 63.68 & 25.29 & 117.55 & 56.02 & 6.75 & 4.09 & ND & 6.02 & 10.83 & 10.07 & ND & 2.72 & 6.14 & 78.80 & 66.40 & 8.72 \\
\hline & W & 79.93 & ND & 120.33 & 55.50 & ND & 6.98 & 14.30 & 4.33 & 15.07 & 11.26 & 5.15 & 11.29 & $\mathrm{ND}$ & 63.50 & 262.33 & 11.58 \\
\hline & Mean & 63.94 & 9.70 & 98.44 & 36.16 & 2.47 & 3.00 & 7.28 & 3.28 & 9.24 & 6.00 & 1.40 & 7.62 & 2.73 & 57.70 & 190.65 & 9.07 \\
\hline & \pm S.D. & 70.48 & 12.19 & 39.31 & 25.14 & 3.17 & 3.16 & 8.40 & 2.55 & 5.18 & 5.51 & 2.51 & 5.81 & 3.20 & 18.59 & 167.76 & 1.79 \\
\hline \multirow{6}{*}{4} & $\mathrm{~S}$ & 58.28 & ND & 127.19 & 3.26 & 0.22 & 0.07 & 23.00 & ND & 3.48 & 2.83 & 0.50 & 11.08 & ND & 35.98 & 396.29 & 10.82 \\
\hline & $\mathrm{S}$ & 73.81 & 12.32 & 54.25 & 18.31 & 4.05 & 1.42 & ND & 6.98 & 3.51 & 2.57 & ND & 1.65 & 3.56 & 48.43 & 40.19 & ND \\
\hline & A & 173.60 & 24.01 & 157.25 & 57.32 & 6.84 & 4.13 & $\mathrm{ND}$ & 16.46 & 11.64 & 10.90 & ND & 2.80 & 6.27 & 81.26 & 92.62 & 8.64 \\
\hline & W & 269.35 & ND & 116.52 & 65.48 & 0.59 & 9.68 & 21.71 & 14.48 & 17.36 & $\mathrm{ND}$ & 5.23 & 12.55 & ND & 67.98 & 273.38 & 12.89 \\
\hline & Mean & 143.76 & 9.08 & 113.80 & 36.09 & 2.92 & 3.83 & 11.18 & 9.48 & 9.00 & 4.08 & 1.43 & 7.02 & 2.46 & 58.41 & 200.62 & 8.09 \\
\hline & \pm S.D. & 98.08 & 11.52 & 43.28 & 30.04 & 3.13 & 4.25 & 12.91 & 7.52 & 6.76 & 4.72 & 2.54 & 5.59 & 3.04 & 20.13 & 164.30 & 5.66 \\
\hline & $\mathrm{S}$ & 72.94 & ND & 167.33 & ND & 1.83 & 9.05 & 29.48 & 2.19 & 9.27 & 7.96 & 9.92 & ND & 1.26 & 52.86 & 395.65 & 1.66 \\
\hline & $\mathrm{S}$ & 77.80 & ND & 32.55 & 23.51 & 4.14 & 0.97 & 0.00 & 9.53 & 3.59 & $\mathrm{ND}$ & 1.06 & 1.68 & $\mathrm{ND}$ & 47.61 & 45.43 & ND \\
\hline & A & 189.61 & ND & 148.55 & 66.51 & 7.87 & 10.00 & 0.00 & 21.61 & 12.53 & $\mathrm{ND}$ & 11.59 & 17.42 & $\mathrm{ND}$ & 90.44 & 202.72 & ND \\
\hline & W & 189.31 & ND & 108.22 & 66.78 & 7.52 & 10.14 & 30.28 & 20.24 & 18.17 & ND & 10.48 & 14.56 & ND & 86.18 & 299.60 & 19.96 \\
\hline & Mean & 132.42 & 14.44 & 114.16 & 39.20 & 5.34 & 7.54 & 14.94 & 13.39 & 10.89 & 7.07 & 8.26 & 8.42 & 0.41 & 69.27 & 235.85 & 5.41 \\
\hline & $\pm \mathrm{SD}$ & 65.90 & 18.37 & 59.73 & 33.11 & 2.87 & 4.40 & 17.25 & 9.21 & 6.10 & 7.78 & 4.84 & 8.85 & 0.59 & 22.15 & 149.39 & 9.73 \\
\hline & $\mathrm{S}$ & 64.28 & ND & 177.16 & 1.50 & 0.06 & 0.03 & ND & 10.91 & 6.11 & 3.07 & 7.66 & 10.91 & ND & 35.61 & 218.09 & ND \\
\hline & $\mathrm{S}$ & 29.90 & ND & 32.55 & 10.51 & 0.42 & 0.07 & ND & 0.70 & 4.40 & 0.99 & 0.01 & 1.68 & $\mathrm{ND}$ & 39.40 & 97.86 & ND \\
\hline & A & 33.89 & 23.11 & 174.25 & 11.94 & 0.51 & 0.08 & 21.94 & 7.26 & ND & $\mathrm{ND}$ & 9.02 & 11.97 & 14.29 & 31.19 & 103.10 & 16.70 \\
\hline & W & 96.42 & 50.91 & 140.55 & 0.57 & 0.41 & ND & 20.98 & 3.08 & ND & ND & 8.64 & 12.44 & ND & 60.93 & 258.70 & 40.19 \\
\hline & Mean & 56.12 & 18.51 & 131.13 & 6.13 & 0.35 & 0.04 & 10.73 & 5.49 & 2.63 & 1.02 & 6.33 & 9.25 & 3.57 & 41.78 & 169.44 & 14.22 \\
\hline & \pm S.D. & 30.90 & 24.19 & 67.78 & 5.92 & 0.19 & 0.03 & 12.39 & 4.52 & 3.11 & 1.44 & 4.25 & 5.08 & 7.14 & 13.19 & 81.30 & 19.01 \\
\hline
\end{tabular}


Table 7. Characteristic values of selected molecular ratios of Pyrogenic and Petrogenic origins of PAHs in P. australis during the study period.

\begin{tabular}{cccccc}
\hline & Phe/Ant & Chry/BaA & Flur/Py & Flur (Flur + Pyr) & LMW/HMW \\
\hline Pyrogenic origin & $<10$ & $<1$ & $>1$ & $>0.5$ & $<1$ \\
Petrogenic origin & $>15$ & $>1$ & $<1$ & $<0.5$ & $>1$ \\
\hline Station & & Results of of the present study & & 0.606 \\
\hline 1 & 2.373 & 0.779 & 1.135 & 0.531 & 0.663 \\
2 & 2.362 & 0.939 & 1.194 & 0.544 & 0.757 \\
3 & 1.065 & 0.773 & 1.636 & 0.62 & 0.75 \\
5 & 1.406 & 0.753 & 1.101 & 0.524 & 0.913 \\
\hline
\end{tabular}

Table 8. Characteristic values of selected molecular ratios of Pyrogenic and Petrogenic origins of PAHs in P. perfoliatus during the study period.

\begin{tabular}{cccccc}
\hline & Phe/Ant & Chry/BaA & Flur/Py & Flur (Flur + Pyr) & LMW/HMW \\
\hline Pyrogenic origin & $<10$ & $<1$ & $>1$ & $>0.5$ & $<1$ \\
Petrogenic origin & $>15$ & $>1$ & $<1$ & $<0.5$ & $>1$ \\
\hline Station & & Results of of the present study & & 0.758 \\
\hline 1 & 1.752 & 0.266 & 1.071 & 0.517 & 0.971 \\
2 & 2.5498 & 0.121 & 1.345 & 0.573 & 0.683 \\
3 & 0.822 & 0.649 & 2.218 & 0.689 & 0.973 \\
5 & 0.763 & 0.453 & 1.179 & 0.541 & 0.821 \\
\hline
\end{tabular}

Table 9. Characteristic values of selected molecular ratios of Pyrogenic and Petrogenic origins of PAHs in P. pectinatus during the study period.

\begin{tabular}{|c|c|c|c|c|c|}
\hline & Phe/Ant & Chry/BaA & Flur/Py & Flur (Flur + Pyr) & LMW/HMW \\
\hline Pyrogenic origin & $<10$ & $<1$ & $>1$ & $>0.5$ & $<1$ \\
\hline Petrogenic origin & $>15$ & $>1$ & $<1$ & $<0.5$ & $>1$ \\
\hline Station & \multicolumn{5}{|c|}{ Results of of the present study } \\
\hline 1 & 8.73 & 0.923 & 1.799 & 0.642 & 0.998 \\
\hline 2 & 4.325 & 0.309 & 1.518 & 0.602 & 0.983 \\
\hline 3 & 0.673 & 0.837 & 1.872 & 0.651 & 0.942 \\
\hline 4 & 2.659 & 0.837 & 1.393 & 0.582 & 0.779 \\
\hline 5 & 3.467 & 0.378 & 2.946 & 0.746 & 0.975 \\
\hline 6 & 0.754 & 0.987 & 1.23 & 0.551 & 0.668 \\
\hline
\end{tabular}


Table 10. Characteristic values of selected molecular ratios of Pyrogenic and Petrogenic origins of PAHs in C. demersumduring the study period.

\begin{tabular}{cccccc}
\hline & Phe/Ant & Chry/BaA & Flur/Py & Flur (Flur + Pyr) & LMW/HMW \\
\hline Pyrogenic origin & $<10$ & $<1$ & $>1$ & $>0.5$ & $<1$ \\
Petrogenic origin & $>15$ & $>1$ & $<1$ & $<0.5$ & $>1$ \\
\hline Station & & Results of the present study & & 0.824 \\
\hline 1 & 3.628 & 0.65 & 1.215 & 0.548 & 0.873 \\
2 & 3.847 & 0.882 & 1.552 & 0.608 & 0.779 \\
4 & 3.044 & 0.883 & 1.482 & 0.597 & 0.994 \\
6 & 3.88 & 0.639 & 1.34 & 0.572 & 0.545 \\
\hline
\end{tabular}

The high PAH concentration in all studied plants, dominated by B(ghi)P indicates the source of PAH pollution is likely to be municipal and medical/pathological waste incinerators [46] and can also be attributed to high levels of automobile emissions (known to contain high levels of it relative to other PAHs) [47] [48]. B(ghi)P is strongly adsorbed to sediment organic matter as its high molecular weight (HMW) renders it resistant to microbial and photo-degradation [49]-[51], so it is expected that greater concentrations of HMW-PAHs are detected during the hot season.

There are significant differences in the concentration of PAHs among studies macrophytes (Figure 2). These differences may be related to the nature of the growth substrate for studied macrophytes, tolerance to environmental conditions for each species, lipid components of plant and surface area that affect the rate of interception and accumulation of PAHs [52]-[54]. Elevated temperature and photic levels (such as during summer) contribute to elevated PAH photo-degradation and can affect the uptake of pollutants in plants as a result, higher concentrations have been recorded during the cold season than the hot season [55].

The PAH origin in the aquatic plants is pyrogenic (Tables 7-10); Al-Hilla River is surrounded by oil fields with flared gas, and crude oil residues and automotive exhaust. Petroleum spills were not evident during the study period. In addition to pyrogenic origin, Al-Taee [56] and Hassan et al. [22] identified petrogenic.

The accumulation of environmental pollution in living organisms is estimated by BCF. The BCF in the aquatic environment is calculated as the ratio of the xenobiotic concentration in the organism to its concentration in the medium [57] [58]. BCF depends on the presence of other organisms or pollutants in the medium and on the contents of lipids in living cells [59]. A greater content of lipids in the organisms causes an increase of the BCF for hydrophobic hydrocarbons and also increases the cytotoxic activity [57].

BCF ranged from 0.05 - 5664.5 for Acp and $\mathrm{B}(\mathrm{a}) \mathrm{P}$ at Sites 4 and 1, respectively for $P$. australis and 0.01 1241 for Acp and B(a)P at Sites 6 and 2 respectively in P. pectinatus. BCF ranges in P. perfoliatus $0.08-1602$ for Ant and B(a)P at Sites 6 and 1, respectively. BCF in C. demersum ranged from $0.16-1141.3$ for B(b)F and B(a)P at Sites 5 and 2, respectively. The BSAF ranged from $3.14-1041$ for Acp and Acpy at Sites 6 and 1, respectively, for $P$. australis, and in $P$. pectinatus ranged from 1.51 for Phe at Site 6 and 976.7 for Ind at Site 6 . The differentiation in results may be due to a combination of plant species, lipid content or surface area in contact with water and sediment [5] [60].

\section{Acknowledgements}

The authors acknowledge the Biology Department, College of Sciences, University of Babylon, and College of Science for Woman, University of Baghdad for their assistance and facilities used to complete this work.

\section{References}

[1] Zhang, C., Li, X., Li, P., Lin, X., Li, Q. and Gong, Z. (2008) Biodegradation of Aged Polycyclic Aromatic Hydrocarbons (PAHs) by Microbial Consortia in Soil and Slurry Phases. Journal of Hazardous Materials, 150, 21-26. 
http://dx.doi.org/10.1016/j.jhazmat.2007.04.040

[2] Thorsen, W., Cope, W.G. and Shea, D. (2004) Bioavailability of PAHs: Effects of Soot Carbon and PAH Source. Environmental Science \& Technology, 38, 2029-2037. http://dx.doi.org/10.1021/es0306056

[3] Al-Azawey, A.N., Salman, J.M. and Hassan, F.H. (2014) Biogeochemical Distribution of Polycyclic Aromatic Hydrocarbons (PAHs), PAHs Biogeochemical Accumulation in Environments. LAP Lambert Academic Publishing, Germany, $222 \mathrm{p}$.

[4] ATSDR (Agency for Toxic Substances and Diseases Regestery) (1996) Toxoprofile by Chemical with NITS, Atlanta.

[5] Salman, J.M., Al-Azawey, A.S.N. and Hassan, F.M. (2014) The Study of Pollution of PAHs (Polycyclic Aromatic Hydrocarbons) in Al-Hilla River, Iraq by Using Bioindicator Freshwater Crab (Sesarma boulengeri Calman). Journal of Life Sciences, 8, 351-357.

[6] Nabatilan, M.M. (2006) Enhanced Degradation of Phenanthrene and Benzo(a) Pyrene in a Field-Contaminated Sediment Inhabited by Ilyodrilus Templetoni: A Microcosm Study. M.Sc. Thesis, University of the Philippines.

[7] Warren, N., Allan, I.J., Carter, J.E., House, W.A. and Parker, A. (2003) Pesticides and Other Micro-Organic Contaminants in Freshwater Sedimentary Environments-A Review. Applied Geochemistry, 18, 159-194. http://dx.doi.org/10.1016/S0883-2927(02)00159-2

[8] Salt, D.E., Smith, R.D. and Raskin, I. (1998) Phytoremediation. Annual Review of Plant Physiology and Plant Molecular Biology, 49, 643-668. http://dx.doi.org/10.1146/annurev.arplant.49.1.643

[9] Prasad, M.N.V., Greger, M. and Aravind, P. (2005) Biogeochemical Cycling of Trace Element by Aquatic and Wetland Plants: Revelance to Phytoremediation. In: Prasad, M.N.V., Kenneh, S. and Naidi, R., Eds., Trace Elements in Environment: Biogeochemistry, Biotechnology and Bioremediation, CRC Press.

[10] Mohan, B.S. and Hosetti, B.B. (1999) Aquatic Plants for Toxicity Assessment. Environmental Research, 81, $259-274$. http://dx.doi.org/10.1006/enrs.1999.3960

[11] Al-Kenzawi, M.A.H. (2009) Seasonal Changes of Nutrient Concentrations in Water of Some Locations in Southern Iraqi Marshes, After Restoration. Baghdad Science Journal, 6, 711-718.

[12] Jones, K.C., Stratford, J.A., Tidridge, P., Waterhouse, K.S. and Johnston, E. (1989) Polynuclear Aromatic Hydrocarbons in an Agricultural Soil: Long-Term Changes in Profile Distribution. Journal of Environmental Pollution, 56, 337351. http://dx.doi.org/10.1016/0269-7491(89)90079-1

[13] Salanitro, J.P., Dorn, P.B., Huesemann, M.H., Moore, K.O., Rhodes, I.A., Rice-Jackson, L.M., Vipond, T.E., Western, M.M. and Wisniewski, H.L. (1997) Crude Oil Hydrocarbon Bioremediation and Soil Ecotoxicity Assessment. Environmental Science and Technology, 31, 1769-1776. http://dx.doi.org/10.1021/es960793i

[14] ASTM (1998) Standard Guide for Conducting Terrestrial Plant Toxicity Tests. In: Annual Book of ASTM Standards, Volume 11.05. E1963-98, American Society for Testing and Materials, West Conshohocken, 20.

[15] Byl, T.D. and Klaine, S.J. (1991) Peroxidase Activity as an Indicator of Sublethal Stress in the Aquatic Plant Hydrilla verticillata (Royal). In: Gorsuch, J.W., Lower, W.R., Wang, W. and Lewis, M.A., Eds., Plants for Toxicity Assessment, Volume 2, American Society for testing and Materiala, STM STP 1115, West Conshohocken, 101-106.

[16] Plewa, M.J. (1991) The Biochemical Basis of the Activation of Promutagens by Plant Cell Systems. In: Gorsuch, J.W., Lower, W.R., Wang, W. and Lewis, M.A., Eds., Plants for Toxicity Assessment, Volume 2, American Society for Testing and Materials, ASTM STP 1115, West Conshohocken, 287-296. http://dx.doi.org/10.1520/stp19523s

[17] Babu, T.S., Marder, J.B., Tripuranthakan, S., Dixon, D.G. and Greenberg, B.M. (2001) Synergistic Effects of a PhotoOxidized PAH and Copper on Photosynthesis and Plant Growth: Evidence That Active Oxygen Formation Is a Mechanism of Copper Toxicity. Environmental Toxicology and Chemistry, 20, 1351-1358. http://dx.doi.org/10.1002/etc.5620200626

[18] Huang, X.D., Dixon, D.G. and Greenberg, B.M. (1993) Impacts of UV Irradiation and Photooxidation on the Toxicity of Polycyclic Aromatic Hydrocarbons to the Higher Plant Lemna gibba L. G3 (Duckweed). Environmental Toxicology and Chemistry, 12, 1067-1077.

[19] Huang, X.D., Glick, B.R. and Greenberg, B.M. (2001) Combining Remediation Techniques for Removal of Persistent Organic Contaminants from Soil. In: Greenberg, B.M., Hull, R.N., Roberts Jr., M.H. and Gensemer, R.W., Eds., Environmental Toxicology and Risk Assessment, Volume 10, American Society for Testing and Materials, West Conshohocken.

[20] Duxbury, C.L., Dixon, D.G. and Greenberg, B.M. (1997) The Effects of Simulated Solar Radiation on the Bioaccumulation of Polycyclic Aromatic Hydrocarbons by the Duckweed Lemna gibba. Environmental Toxicology and Chemistry, 16, 1739-1748. http://dx.doi.org/10.1002/etc.5620160824

[21] Thomas, G., Sweetman, A.J., Ockenden, W.A., Mackay, D. and Jones, K.C. (1998) Air-Pasture Transfer of PCBs. Environmental Science and Technology, 32, 936-942. http://dx.doi.org/10.1021/es970761a 
[22] Hassan, F.M., Salman, J.M., Al-Azawey, A.S.N., Al-Ansari, N. and Kutsson, S. (2014) Quality, Quantity and Origin of PAHs (Polycyclic Aromatic Hydrocarbons) in Lotic Ecosystem of Al-Hilla River, Iraq. Journal of Civil Engineering and Architecture, 8, 1026-1038.

[23] Kayal, S. and Conell, D.W. (1995) Polycyclic Aromatic Hydrocarbons in Biota from the Brisbane River Estuary, Australia. Estuarine, 40, 475-493. http://dx.doi.org/10.1006/ecss.1995.0033

[24] Malhat, F.M., Nasr, I.N., Arief, M.H. and Abdel-Aleem, A.H. (2010) Polycyclic Aromatic Hydrocarbons (PAHs) in Aquatic Environment at El Menofiya Governorate. Egypt Journal of Applied Science Research, 6, 13-21.

[25] Berset, J.D., Ejem, M., Holzer, R. and Lischer, P. (1999) Comparison of Different Drying, Extraction and Detection Techniques for the Determination of Priority Polycyclic Aromatic Hydrocarbons in Background Contaminated Soil Samples. Analytica Chimica Acta, 383, 263-275. http://dx.doi.org/10.1016/S0003-2670(98)00817-4

[26] Maskaoui, K., Zhou, J.L., Hong, H.S. and Zhang, Z.L. (2002) Contamination by Polycyclic Aromatic Hydrocarbons in the Jiulong River Estuary and Western Xiamen Sea, China. Environmental Pollution, 118, 109-122. http://dx.doi.org/10.1016/S0269-7491(01)00208-1

[27] Sanders, M., Sivertsen, S. and Scott, G. (2002) Origin and Distribution of Polycyclic Aromatic Hydrocarbon in Superficial Sediments from the Savannah River. Archives of Environmental Contamination and Toxicology, 43, 438-448. http://dx.doi.org/10.1007/s00244-002-1232-1

[28] Doong, R. and Lin, Y. (2004) Characterization and Distribution of Polycyclic Aromatic Hydrocarbon Contaminations in Surface Sediment and Water from Gao-Ping River, Taiwan. Water Research, 38, 1733-1744. http://dx.doi.org/10.1016/j.watres.2003.12.042

[29] Cal-EPA (1996) Soil Screening Guidance: User’s Guide. Second Edition, Technical Support Document for Describing Available Cancer Potency Factors, Office of Environmental Emergency and Remedial Response, US Environmental Protection Agency, Washington DC.

[30] Ikhajiagbe, B., Anoliefo, G.O., Omoregbee, O. and Osigbemhe, P. (2014) Changes in the Intrinsic Qualities of a Naturally Attenuated Waste Engine Oil Polluted Soil after Exposure to Different Periods of Heat Shock. Resources and Environment, 4, 45-53.

[31] EPA (1993) Provisional Guidance for Quantitative Risk Assessment of Polycyclic Aromatic Hydrocarbons. EPA/600/ R-93/089, US Environmental Protection Agency, Cincinnati.

[32] Qiao, M., Wang, C., Huang, S., Wang, D. and Wang, Z. (2006) Composition, Sources, and Potential Toxicological Significance of PAHs in the Surface Sediments of the Meiliang Bay, Taihu Lake, China. Environment International, 32 , 28-33. http://dx.doi.org/10.1016/j.envint.2005.04.005

[33] Sirece, M.A., Marty, J.C., Saliot, A., Aparicio, X., Grimalt, J. and Albaiges, J. (1987) Aliphatic and Aromatic Hydrocarbons in Different Sized Aerosols over Mediterranean Sea: Occurrence and Origin. Atmosphere Environment, 21, 2247-2259. http://dx.doi.org/10.1016/0004-6981(87)90356-8

[34] Markert, B.A., Breure, A.M. and Zechmeister, H.G., Eds. (2003) Bioindicators and Biomonitors. Elsevier, Oxford, 977p.

[35] Habeeb, M.A., Al-Bermani, A.K. and Salman, J.M. (2015) Environmental Study of Water Quality and Some Heavy Metals in Water, Sediment and Aquatic Macrophytas in Lotic Ecosystem, Iraq. Mesopotamia Environment Journal, 1, 66-84.

[36] Al-Taee, M.M.S. and Witwit, R.T.A. (2015) Evaluation of Antioxidant Enzyme Activities during Phytoremediation of Textile Waste Water Exposed of Structurally Different Dyes by Some Aquatic Plants. Mesopotamia Environment Journal, 1, 35-65.

[37] Prasad, M.N.V. (2008) Aquatic Plants for Phytotechnology. In: Prasad, M.N.V., Ed., Metals in the Environment: Analysis by Biodiversity, Marcel Dekker Inc., New York, 259.

[38] Adriano, D.C., Wenzel, W.W., Vangronsveld, J. and Bolan, N.S. (2004) Role of Assisted Natural Remediation in Environmental Cleanup. Geoderma, 122, 121-142. http://dx.doi.org/10.1016/j.geoderma.2004.01.003

[39] Brix, H. and Schierup, H.H. (1989) The Use of Macrophytes in Water-Pollution Control. Ambio, 18, 100-107.

[40] Kiedrzynska, E., Wagner, I. and Zalewski, M. (2008) Quantification of Phosphorus Retention Efficiency by Floodplain Vegetation and a Management Strategy for a Eutrophic Reservoir Restoration. Ecological Engineering, 33, 15-25. http://dx.doi.org/10.1016/j.ecoleng.2007.10.010

[41] Ulrich, K.E. and Burton, T.M. (1985) The Effects of Nitrate, Phosphate, and Potassium Fertilization on Growth and Nutrient Uptake Patterns of Phragmites australis. Aquatic Botany, 21, 53-62. http://dx.doi.org/10.1016/0304-3770(85)90095-6

[42] Greenway, M. and Woolley, A. (1999) Constructed Wetlands in Queensland: Performance Efficiency and Nutrient Bioaccumulation. Ecological Engineering, 12, 39-55. http://dx.doi.org/10.1016/S0925-8574(98)00053-6

[43] Ruiz, M. and Velasco, J. (2010) Nutrient Bioaccumulation in Phragmites australis: Management Tool for Reduction of 
Pollution in the Mar Menor. Water, Air, \& Soil Pollution, 205, 173-185. http://dx.doi.org/10.1007/s11270-009-0064-2

[44] Aravind, P. and Prasad, M.N.V. (2005) Zinc Mediated Protection to the Conformation of Carbonic Anhydrase in Cadmium Exposed Ceratophyllum demersum L. Plant Science, 69, 245-254. http://dx.doi.org/10.1016/j.plantsci.2005.03.028

[45] Esteves, B.D.S. and Suzuki, M.S. (2010) Limnological Variables and Nutritional Content of Submerged Aquatic Macrophytes in a Tropical Lagoon. Acta Limnologica Brasiliensia, 22, 187-198. http://dx.doi.org/10.1590/S2179-975X2010000200008

[46] Williams, R., Meares, J. and Brooks, L. (1994) Priority Pollutant PAH Analysis of Incinerator Emission Particles Using HPLC and Optimized Fluorescence. International Journal of Environmental Analytical Chemistry, 54, $299-314$.

[47] Rogge, W.F., Hildemann, L.M. and Mazurek, M.A. (1993) Sources of Fine Organic Aerosol. 2. Noncatalyst and Catalyst-Equipped Automobiles and Heavy-Duty Diesel Trucks. Environmental Science \& Technology, 27, 636-651. http://dx.doi.org/10.1021/es00041a007

[48] Santodonato, J. (1981) Polycyclic Organic Matter. Journal of Environmental Pathology \& Toxicology, 5, 1-364.

[49] EHC-202 (Environmental Health Criteria) (1998) Selected Non-Heterocyclic Aromatic Hydrocarbon. International Program on Chemical Safety.

[50] Eisler, R. (1987) Polycyclic Aromatic Hydrocarbon Hazards to Fish, Wildlife and Invertebrates: A Synoptic Review. US Fish and Wildlife Service Biological Report, 85, 81.

[51] Irwin, R.J. (1997) Environmental Contaminants Encyclopedia, PAHs Entry. National Park Service.

[52] Sims, R.C. and Overcash, M.R. (1983) Fate of Polynuclear Aromatic Compounds (PNAs) in Soil-Plant Systems. Residue Reviews, 88, 1-68. http://dx.doi.org/10.1007/978-1-4612-5569-7_1

[53] Thomas, W. (1984) Statistical Models for the Calculation of PAH, Chlorinated Hydrocarbons and Trace Metals in Epiphytic Hypnum cupressiforme. Water, Air, \& Soil Pollution, 22, 351-371. http://dx.doi.org/10.1007/BF00282607

[54] Edwards, N.T. (1988) Assimilation and Metabolism of Polycyclic Aromatic Hydrocarbons by Vegetation-An Approach to This Controversial Issue and Suggestions for Future Research. In: Cook, M. and Dennis, A.J., Eds., Polycyclic Aromatic Hydrocarbons: A Decade of Progress, Battelle Press, Columbus, 211-229.

[55] Alfani, A., Nicola, F.D., Maisto, G. and Pratim, M.V. (2005) Long-Term PAH Accumulation after Bud Break in Qurrcus ilex L. Leaves in Polluted Environment. Atmospheric Environment, 39, 307-314. http://dx.doi.org/10.1016/j.atmosenv.2004.09.001

[56] Al-Taee, M.M.S. (2010) Distribution and Source of Polycyclic Aromatic Hydrocarbons (PAHs) in Surficial Sediment from Shatt Al-Hilla River/Iraq. Marsh Bulletin, 5, 43-55.

[57] Fisher, S.W. (1995) Mechanisms of Bioaccumulation in Aquatic Systems. Reviews of Environmental Contamination and Toxicology, 142, 87-117. http://dx.doi.org/10.1007/978-1-4612-4252-9_4

[58] Sheedy, B.R., Mattson, V.R., Cox, J.S., Kosian, P.A., Phipps, G.L. and Ankley, G.T. (1998) Bioconcentration of Polycyclic Aromatic Hydrocarbons by the Freshwater Oligochaete Lumbriculus variegatus. Chemosphere, 36, 30-61. http://dx.doi.org/10.1016/S0045-6535(98)00007-1

[59] Lee, R.R., Sauerheber, R. and Dobbs, G.H. (1972) Uptake, Metabolism and Discharge of Polycyclic Aromatic Hydrocarbons by Marine Fish. Marine Biology, 17, 201-208. http://dx.doi.org/10.1007/BF00366294

[60] Wild, S.R. and Jones, K.C. (1991) Studies of the Polynuclear Aromatic Hydrocarbon Content of Carrots (Daucus carota). Chemosphere, 23, 243-251. http://dx.doi.org/10.1016/0045-6535(91)90110-Y 